\title{
Receptive Field Plasticity Profoundly Alters the Cutaneous Parallel Fiber Synaptic Input to Cerebellar Interneurons In Vivo
}

\author{
Henrik Jörntell and Carl-Fredrik Ekerot \\ Department of Physiological Sciences, Section for Neurophysiology, SE-221 84 Lund, Sweden
}

\begin{abstract}
The cutaneous parallel fiber (PF) receptive fields of cerebellar stellate and basket cells in the cerebellar $\mathrm{C} 3$ zone in vivo are normally very small but can be dramatically enlarged by climbing fiber (CF)-dependent plasticity. To analyze the effects of this receptive field plasticity, we present for the first time whole-cell patch-clamp recordings from these interneurons during natural and electrical activation of cutaneously driven synaptic input. In "naive" interneurons, peripheral input nearly exclusively activated a few (two to eight) large PF EPSPs from a specific small skin area that overlapped the receptive field of the local CF input. After conjunctive PF and CF stimulation, numerous small and large EPSPs and ramp-like depolarizations could be activated from the entire forelimb skin. These findings therefore confirm previous suggestions that conjunctive $\mathrm{PF}$ and $\mathrm{CF}$ activation leads to a long-lasting potentiation of PF synaptic input to interneurons. The $\mathrm{CF}$ response, which is crucial for the induction of the PF synaptic potentiation, was strong but variable and very different from the conventional EPSPs evoked by PFs.
\end{abstract}

Key words: stellate cells; basket cells; parallel fibers; climbing fibers; plasticity; cerebellum

\section{Introduction}

Cerebellar molecular layer interneurons are believed to receive a few hundred excitatory parallel fiber (PF) synapses (Palay and Chan-Palay, 1974; Carter and Regehr, 2002) as well as one or a few climbing fiber (CF) synapses (Palay and Chan-Palay, 1974; Sugihara et al., 1999). These interneurons also receive inhibitory input from other interneurons but not from Purkinje cells (Eccles et al., 1967; Palay and Chan-Palay, 1974; De Camilli et al., 1984) (see Fig. 1A). In the cerebellar C3 zone, a main input is from mossy fibers (MFs), which transmit events fast and faithfully from small cutaneous receptive fields (Garwicz et al., 1998). MFs activate granule cells, the efferent PFs of which are several millimeters long, and ensure that any neuron within the C3 zone can sample MF input from a large volume of cortex (Eccles et al., 1967; Brand et al., 1976). However, extracellular recordings revealed that along a beam of PFs, interneurons have small and distinct but widely different cutaneous receptive fields that resembled those of single MFs terminating in the granule layer directly below the cell (Ekerot and Jörntell, 2001, 2003). These findings suggested that in normal adult cerebella, each interneuron has selected a privileged set of the available PFs that provide a particularly strong influence on its spike firing. Because the receptive fields of interneurons were nearly identical to the recep-

Received May 13, 2003; revised Aug. 21, 2003; accepted Aug. 26, 2003.

This work was supported by the Swedish Medical Research Council (project number 8291), the Segerfalk Foundation, the Swedish Medical Society, Crafoords Stiftelse, Magnus Bergwalls Stiftelse, and the Swedish Society for Medical Research. We also thank our technician, Kersti Larsson, for her extensive work during the processing of stained neurons.

Correspondence should be addressed to Henrik Jörntell, Department of Physiological Sciences, Section for Neurophysiology, Biomedical Center F10, Tornavägen 10, SE-22184 Lund, Sweden. E-mail: henrik.jorntell@mphy.lu.se. Copyright $\odot 2003$ Society for Neuroscience $\quad$ 0270-6474/03/239620-12\$15.00/0 tive field of the local CF, we suggested that the role of the CF input to the interneuron may be to strengthen the PF synapses forming the privileged set, leaving all other PF synapses on the cell strongly depressed or electrically silent (Ekerot and Jörntell, 2001, 2003). Indeed, it was subsequently shown that the depressed cutaneous $\mathrm{PF}$ input is possible to recruit using PF burst stimulation in conjunction with CF activation. This potentiation of cutaneous PF input remained for several hours (Jörntell and Ekerot, 2002). Furthermore, the recruited skin input could be silenced again using the same PF burst stimulation but omitting the $\mathrm{CF}$ activation.

Here we wanted to perform intracellular recordings in the same in vivo preparation to understand more fully the integration of cutaneous synaptic input in naive interneurons as well as after potentiation of the cutaneous PF input. By being able to apply the whole-cell patch-clamp recording technique, which was originally devised for the in vitro preparation (Hamill et al., 1981), in our in vivo preparation we could obtain high-quality intracellular recordings from these small interneurons and perform the desired analysis.

\section{Materials and Methods}

Adult cats were prepared as described previously (Ekerot and Jörntell, 2001; Jörntell and Ekerot, 2002). Briefly, after an initial anesthesia with propofol (Diprivan, Zeneca, Macclesfield Cheshire, UK), the animals were decerebrated at the intercollicular level and mounted in a stereotaxic frame. The animals were artificially ventilated and the endexpiratory $\mathrm{CO}_{2}$, blood pressure, and rectal temperature were monitored continuously and maintained within physiological limits. Drainage of CSF and pneumothorax and the clamping of the spinal processes of a few cervical and lumbar vertebral bodies served to increase the mechanical 
stability of the preparation. The postoperative EEG was characterized by periods of low-frequency, large-amplitude waves.

Recordings and stimulation. The initial delineation of the forelimb area of the $\mathrm{C} 3$ zone in the cerebellar anterior lobe and the continuous monitoring of the general condition in the sensitive mossy fiber to granule cell to parallel fiber pathway were performed as described previously (Ekerot and Jörntell, 2001; Jörntell and Ekerot, 2002).

Two coiled silver wires, forming an outer and an inner ring, respectively, were put on the surface of the cerebellar cortex to stabilize the recording area. One to three homemade tungsten-in-glass microelectrodes (exposed tips 50-150 $\mu \mathrm{m}$ ) were inserted in the superficial cortical sheet for stimulation of PFs. A surface electrode or an intracortical microelectrode was left in place within $0.3 \mathrm{~mm}$ from the area in which patch-clamp recordings were made to study the correlation between the intracellular and extracellular activities. Another tungsten microelectrode was inserted near the primary fissure $\sim 1 \mathrm{~mm}$ from the midline to reach the contralateral inferior olive. All stimulation through tungsten electrodes was made with pulse widths of $0.2 \mathrm{msec}$. To have as precise activation of CFs as possible, the CF responses evoked from the microelectrode advanced into the inferior olive were monitored continuously by a surface electrode located at the site from which patch-clamp recordings were made subsequently.

Some of the interneurons were recorded in PF beams that had been subjected to the stimulation protocol that we have shown previously to induce dramatic receptive field enlargements in interneurons recorded extracellularly (Jörntell and Ekerot, 2002). The protocol consisted of electrical burst stimulation of PFs (15 pulses at $100 \mathrm{~Hz}$, at $50-150 \mu \mathrm{A}$ ) and a simultaneous single electrical shock in the inferior olive and was repeated at $0.33 \mathrm{~Hz}$ for $10 \mathrm{~min}$. Note that because of the length of the stimulation protocol and the time it takes for the effects to develop (at least 5-10 min) (Jörntell and Ekerot, 2002), we were unable to follow a single interneuron before and after the stimulation protocol. In normal interneurons, the receptive field is always nearly completely overlapping that of the local CF (Ekerot and Jörntell, 2001, 2003; Jörntell and Ekerot, 2002). Therefore, in interneurons with potentiated PF input, the recorded local CF receptive field is referred to as the original receptive field of the interneuron, whereas other skin areas providing input are referred to as induced receptive fields.
To construct peristimulus histograms of neuronal activity evoked from the skin during manual stimulation, we used a strain gauge device mounted on the index finger of the investigator. The signal of the strain gauge device was used mainly as a trigger signal and as a general indication that approximately the same amount of force was applied during stimulations. Because we used rapid strokes by the index finger as stimulation, the strain gauge device could have slightly different angles at the time of skin contact; therefore, a detailed analysis of the force measured by the strain gauge was not meaningful.

Patch-clamp electrodes were pulled to 5-15 $\mathrm{M} \Omega$ from borosilicate glass capillaries on a vertical puller (Narishige NN-850; Narishige, Tokyo, Japan) and filled with a solution containing (in $\mathrm{mm}$ ): 135 K-gluconate, $7 \mathrm{KCl}, 0.1 \mathrm{CaCl}_{2}, 10 \mathrm{HEPES}$, and $2 \mathrm{Mg}$-ATP. The solution was titrated to $\mathrm{pH} 7.35-7.40$ using $\mathrm{KOH}$. In some cases, biocytin (10-60 mм) (Sigma, St Louis, MO) was added to the internal solution. After the experiments, the cats were killed by barbiturate and then perfused by $4 \%$ paraformaldehyde. After incubation in PBS overnight, the tissue was sectioned approximately parasagittally at $60 \mu \mathrm{m}$ in a freezing microtome. Biocytin staining was processed using a standard ABC kit with Vector SG (Vectastain Elite ABC kit and Vector SG; Vector Laboratories, Burlingame, CA).

Patch-clamp electrodes were inserted so that they were perpendicular to the cortical surface. A microdriver step counter was reset on electrical contact with the brain and subsequently used to keep track of the recording depth. A homemade electrometer amplifier was used for patch-clamp recordings. The amplifier used an Analog Devices AD 549LH operational amplifier as input stage and had the following characteristics: input capacitance, $<2 \mathrm{pF}$; input resistance, $>1 \mathrm{~T} \Omega$; input current, $<1 \mathrm{pA}$. All recordings were digitized continuously at $20-40 \mathrm{kHz}$ and stored on a computer disk by homemade software. The electrode signal was monitored continuously through loudspeakers, by display on oscilloscopes, by a digital video projector, and on a computer screen. Whole-cell patchclamp recordings were obtained as follows: the patch-clamp electrode was advanced under positive pressure at the tip, and a $10 \mathrm{msec}$ rectangular current pulse at $1 \mathrm{nA}$ was used to monitor the electrode resistance. When a sudden substantial increase in the electrode resistance occurred, a hyperpolarizing current of $0.1-0.9 \mathrm{nA}$ and a negative pressure were applied to establish a gigaohm seal $(0.5-10 \mathrm{G} \Omega)$ between the electrode

Table 1. Properties of spontaneous and evoked synaptic responses

\begin{tabular}{|c|c|c|}
\hline & Normal interneurons $(n=8)$ & After conjunctive CF-PF stimulation $(n=4)$ \\
\hline \multicolumn{3}{|l|}{ Properties of synaptic potentials ${ }^{a}$} \\
\hline \multicolumn{3}{|l|}{ EPSP properties } \\
\hline Maximal amplitude ( $-55 \mathrm{mV})$ & $5.9 \pm 0.47 \mathrm{mV}$ & $5.6 \pm 0.38 \mathrm{mV}$ \\
\hline Time-to-peak & $0.98 \pm 0.13 \mathrm{msec}$ & $1.0 \pm 0.12 \mathrm{msec}$ \\
\hline Half-decay time & $4.3 \pm 0.29 \mathrm{msec}$ & $4.2 \pm 0.27 \mathrm{msec}$ \\
\hline Frequency & $36 \pm 15 \mathrm{~Hz}$ & $54 \pm 11 \mathrm{~Hz}$ \\
\hline \multicolumn{3}{|l|}{ IPSP properties } \\
\hline Maximal amplitude ( $-55 \mathrm{mV})$ & $4.6 \pm 0.47 \mathrm{mV}$ & $5.2 \pm 0.42 \mathrm{mV}$ \\
\hline Time-to-peak & $2.0 \pm 0.36 \mathrm{msec}$ & $2.1 \pm 0.38 \mathrm{msec}$ \\
\hline Half-decay time & $9.8 \pm 1.3 \mathrm{msec}$ & $10.5 \pm 4.1 \mathrm{msec}$ \\
\hline Frequency & $20 \pm 5 \mathrm{~Hz}$ & $33 \pm 9 \mathrm{~Hz}$ \\
\hline \multicolumn{3}{|c|}{ (F-EPSP properties (averages at $-60 \mathrm{mV}$ ) } \\
\hline Amplitude & $2.8 \pm 0.89 \mathrm{mV}$ & $2.2 \pm 0.67 \mathrm{mV}$ \\
\hline Time-to-peak & $6.8 \pm 0.78 \mathrm{msec}$ & $6.2 \pm 1.1 \mathrm{msec}$ \\
\hline \multirow[t]{2}{*}{ Half-decay time } & $9.9 \pm 3.0 \mathrm{msec}$ & $9.2 \pm 3.6 \mathrm{msec}$ \\
\hline & Normal interneurons $(n=4)$ & After conjunctive CF-PF stimulation $(n=4)$ \\
\hline \multicolumn{3}{|c|}{ Responses to manual cutaneous stimulation ${ }^{b}$} \\
\hline CF receptive field ( 1 per neuron) & $0.43 \pm 0.30 \mathrm{mV}(n=61)$ & $0.52 \pm 0.28 \mathrm{mV}(n=55)$ \\
\hline 0ther skin areas ( $2-10$ per neuron) & $0.01 \pm 0.076 \mathrm{mV}(n=201)$ & $0.51 \pm 0.33 \mathrm{mV}(n=210)$ \\
\hline Pre-stimulus baseline ( $50 \mathrm{msec}$ ) & $0.38 \pm 0.083 \mathrm{mV}$ & $0.67 \pm 0.14 \mathrm{mV}$ \\
\hline
\end{tabular}

aPSP properties were analyzed in 12 different neurons ( 4 of which were recorded after conjunctive PF-CF stimulation) for PSPs that appeared uncontaminated by preceding or superimposed PSPs. For each neuron, $10-20$ PSPs of various amplitudes were selected for calculation of PSP kinetics. The rates of spontaneous PSP activity were calculated for all PSPs with an absolute peak amplitude of $\geq 1.3 \mathrm{mV}$. CF-EPSP properties were analyzed in averaged responses (10-20 olivary stimulations each) recorded in the same 12 neurons.

${ }^{b}$ The initial $50 \mathrm{msec}$ of the responses evoked by manual cutaneous stimulation was rectified. The average voltage deflection for these $50 \mathrm{msec}$ was calculated for each stimulation. The voltage deflection of the preceding $50 \mathrm{msec}$ was then subtracted. For each neuron, each skin site was stimulated $8-27$ times. Notably, stimulation of skin areas outside the CF receptive field, which normally did not evoke any response, was induced to do so after conjunctive PF-CF stimulation. All data are shown as means \pm SD. 
A



B

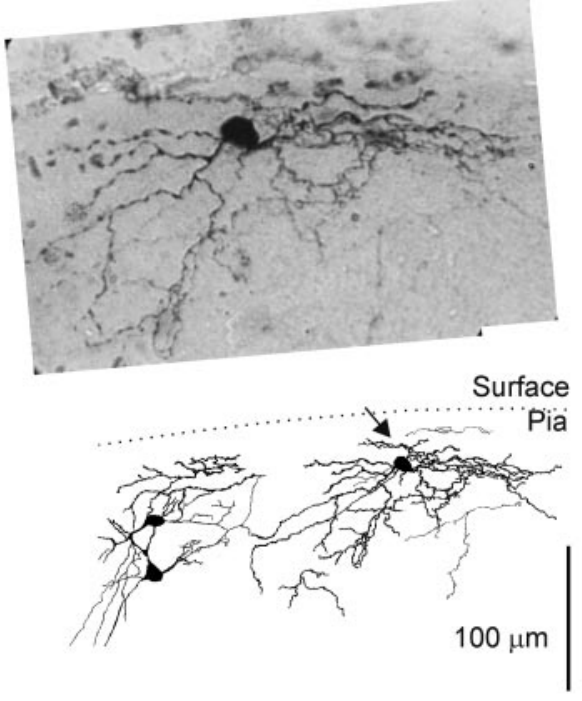

C

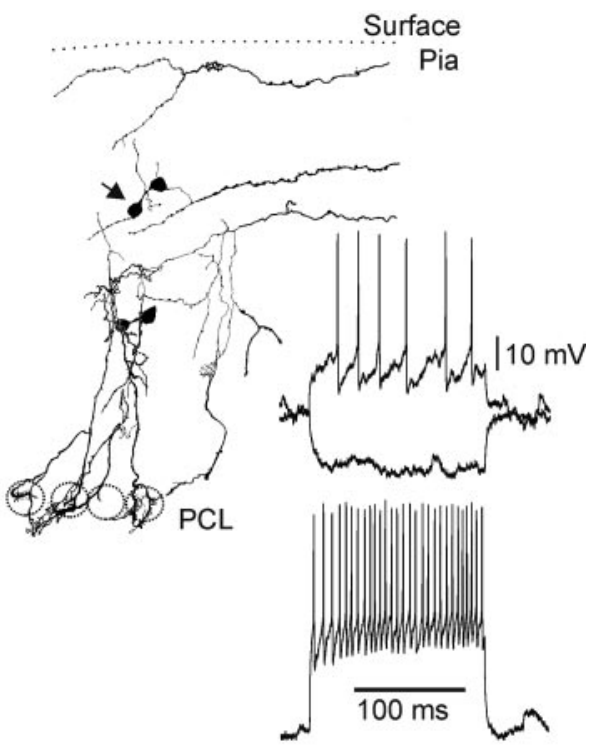

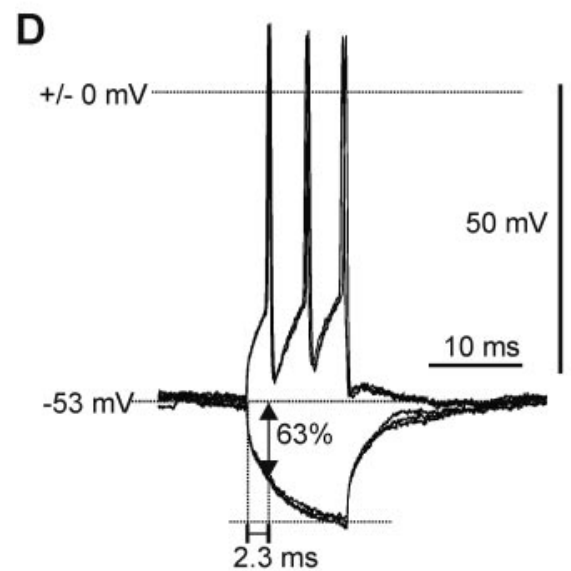

E



Figure 1. Basic properties of molecular layer interneurons. A, Schematic circuitindicating the neuronal connections of molecular layer interneurons (Int). The efferent inhibition onto Purkinje cells (PC) and the afferent inhibitory input from other interneurons are indicated as filled triangles; the other connections are excitatory. The CF innervation of interneurons is through short, thin collaterals of the (F arborization covering the dendritic tree of adjacent Purkinje cells (Sugihara et al., 1999). grc, Granule cells. B, Photomicrograph and drawing tube reconstruction of a superficial interneuron stained by biocytin. C, Drawing tube reconstruction of another biocytin-stained interneuron recorded at mid-depth in the molecular layer (arrow). Note the descending axon branches forming basket-like structures around and beneath the Purkinje cell layer (PCL), suggesting that also mid-molecular layer interneurons can belong to the basket cell type [as shown previously by Sultan and Bower (1998)]. Inset to the right shows the responses of the cell to rectangular current injections of \pm 60 and $+150 \mathrm{pA}$. In $B$ and $C$, the most densely stained cell body is indicated by an arrow; however, cell bodies of several neighboring interneurons were stained, probably as a result of communications through gap junctions between these cells (Mann-Metzer and Yarom, 1999). Parts of the stained neurons appear to have been lost because of their location between two sections. The full extent of axons is not shown. $D$, Membrane responses to current steps of $\pm 420 \mathrm{pA}$ in another interneuron. $E$, In another interneuron, membrane responses to current injections of $\pm 120 \mathrm{pA}$ (left) and spontaneous action potentials at $0 \mathrm{pA}$ bias current (right). Spontaneous action potentials were preceded by large EPSP-like events (bottom right). Membrane responses in $D$ and $E$ are from normal interneurons, whereas the neuron in $C$ was recorded after the standard protocol with conjunctive PF burst and CF stimulation.

and the recorded cell. Once established, brief increases in negative pressure were used to break the seal and gain contact with the interior of the cell. The junction potential between the electrode solution and the extracellular environment of the cerebellum was $\sim 5-7 \mathrm{mV}$ in two experiments but was not compensated for in the displays.

Analysis of EPSPs and IPSPs. The analysis performed in this paper, except when stated otherwise, was made in interneurons that were prevented from firing spontaneously by using mild hyperpolarizing currents of $0.03-0.2 \mathrm{nA}$. The amount of bias current needed to prevent cells from firing spontaneously, the maximal EPSP amplitudes when the membrane potential was just subthreshold for spontaneous firing, and the amplitudes of occasional evoked spikes were monitored continuously to check the quality of the recording. Any substantial deviations from the maximal values for the neuron and the normal values for the population were taken as a sign of poor recording conditions, and such neurons-periods were excluded from the analysis. The plateau phase of the membrane response to hyperpolarizing rectangular current steps was used for calculation of the input resistance. The time to reach a level corresponding to $63 \%$ of the difference between the holding potential and the plateau level was taken as the time constant. Homemade software was used to identify EPSP- and IPSP-like events. Identification of events and measurements of peak amplitudes were made semiautomatically: the software found and displayed events, and the user accepted or rejected and identified the peak amplitude of the event. This semiautomatic procedure was checked manually to accurately find $90-100 \%$ of all EPSPs and IPSPs that exceeded $\sim 1.3 \mathrm{mV}$ in peak amplitude.

The experimental procedures were approved in advance by the local Swedish Animal Research Ethics Committee.

\section{Results}

The present analysis was performed in 31 molecular layer interneurons from which high-quality intracellular recordings lasting 
5-60 min were obtained. Six of these interneurons were recorded after a standard conjunctive PF burst-CF stimulation protocol (see Materials and Methods). Except where stated otherwise, for the basic properties and responses evoked by PF and CF stimulation there were no differences between the two groups of interneurons (Table 1). Molecular layer interneurons were recorded in the superficial molecular layer at recording depths of $0.04-$ $0.31 \mathrm{~mm}$ from the pial surface, which was confirmed to be above the Purkinje cell layer by extracellular recordings. Some of the interneurons were identified morphologically (Fig. $1 B, C$ ). Interneurons could be connected morphologically with other nearby interneurons, and spikelets of the type that has been interpreted as electrotonically conducted spikes from connected interneurons in vitro (Galaretta and Hestrin, 1999, 2001; Gibson et al.,
1999; Mann-Metzer and Yarom, 1999) were sometimes observed, although they were limited to very small events $(\leq 1.0$ $\mathrm{mV}$ ). In addition, interneurons were characterized by large, fast spikes and the absence of large, broad depolarizations typical for spontaneous CF responses in Purkinje cell dendrites (Llinas and Sugimori, 1980; Ekerot and Oscarsson, 1981; Callaway et al., 1995). The spike activity was readily controlled by small currents injected intracellularly (Fig. $1 C-E$ ). The input resistances were $139 \pm 46 \mathrm{M} \Omega($ mean $\pm \mathrm{SD} ; n=17$; range, $52-330 \mathrm{M} \Omega)$, which is $1 / 5-1 / 30$ of the values reported for cerebellar molecular layer interneurons in vitro (Häusser and Clarke, 1997; Mann-Metzer and Yarom, 1999; Kreitzer et al., 2002; Chavas and Marty, 2003). At least in part, this big difference in input resistance might be explained by the much higher background activity of synaptic
A

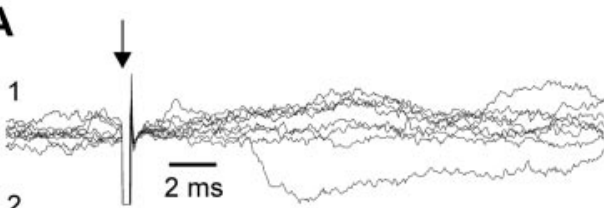

2

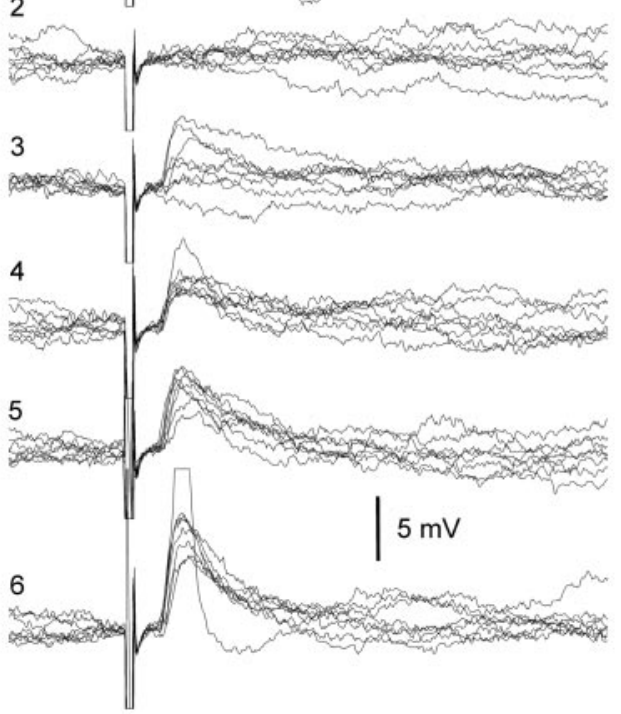

B

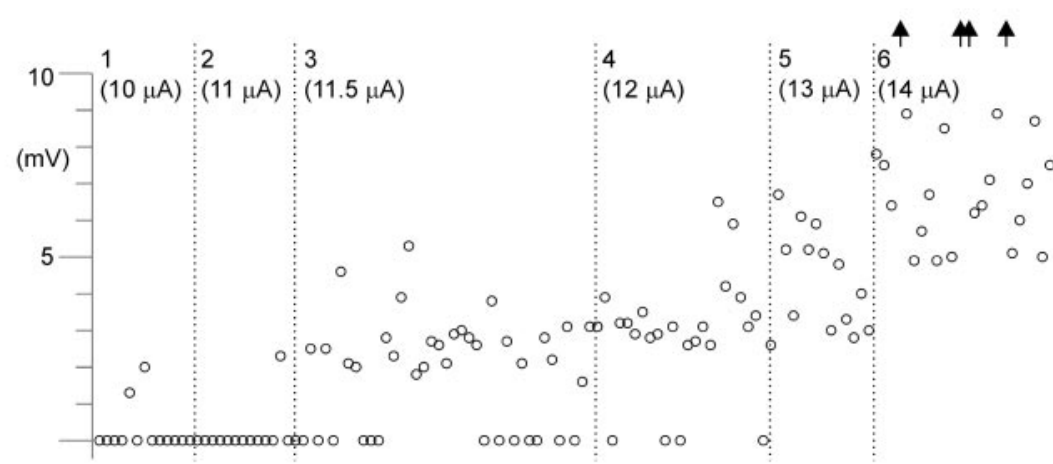

C
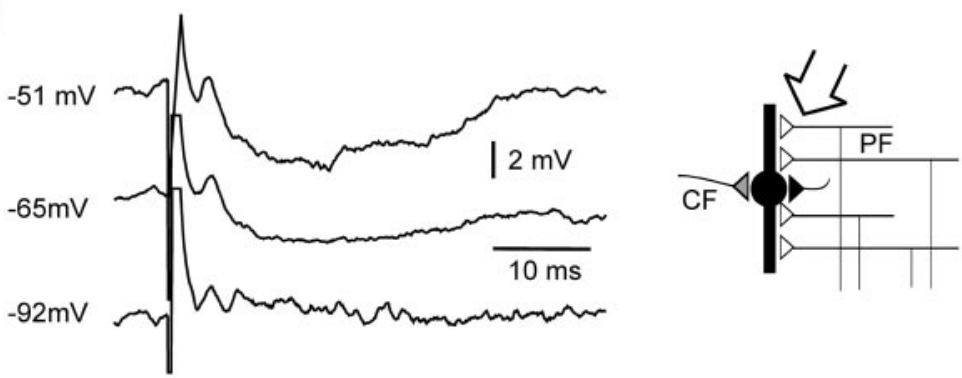

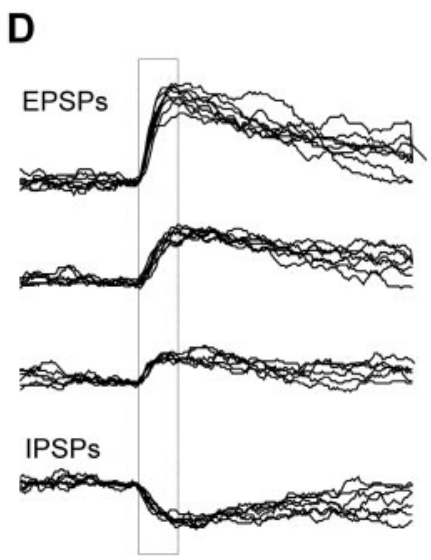

$\mathbf{E}$

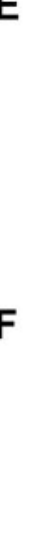

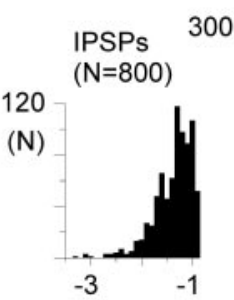
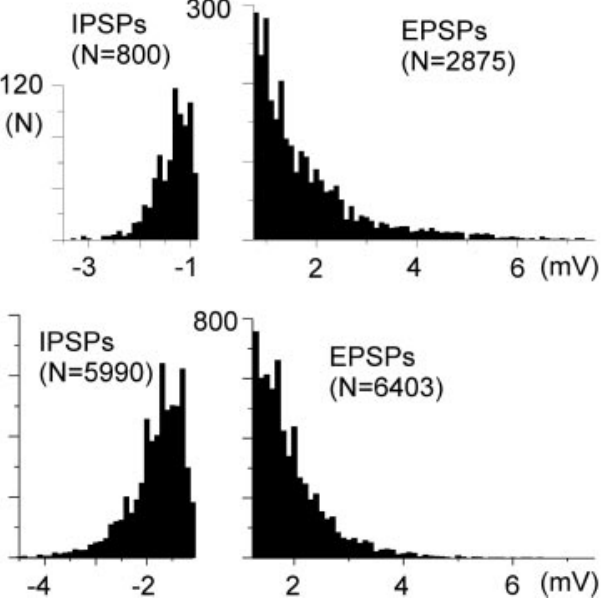

G

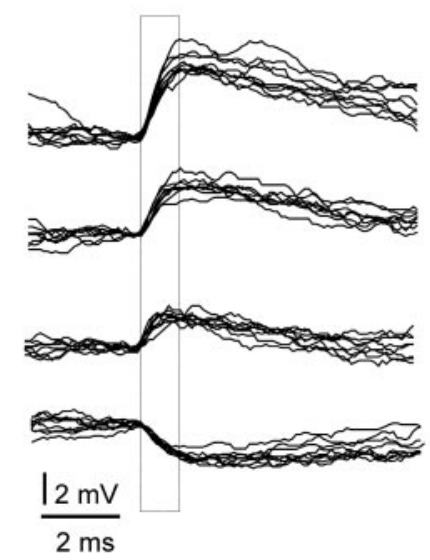

Figure 2. PF-evoked and spontaneous putative EPSPs and IPSPS. A, Raw data sweeps obtained during electrical PF stimulation (arrow) around response threshold. B, Plot of EPSP amplitudes evoked at different PF stimulation intensities. Arrows indicate that the PF stimulation evoked a spike. C, In another cell, off-beam PF stimulation evoked an IPSP, which reversed polarity at a membrane potential of -85 to $-90 \mathrm{mV}$. Note PF volley preceding the IPSP. Inset, Schematic circuit diagram highlighting the PF synapses. D, Spontaneous putative EPSPs and IPSPs. Narrow box encloses $1 \mathrm{msec}$ after PSP onset. E, Amplitude frequency histograms of spontaneous EPSPs and IPSPs. F, G, Display as in D and Ebut for an interneuron recorded after conjunctive PF-CF stimulation. 
A

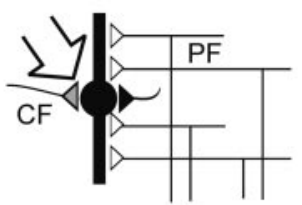

B
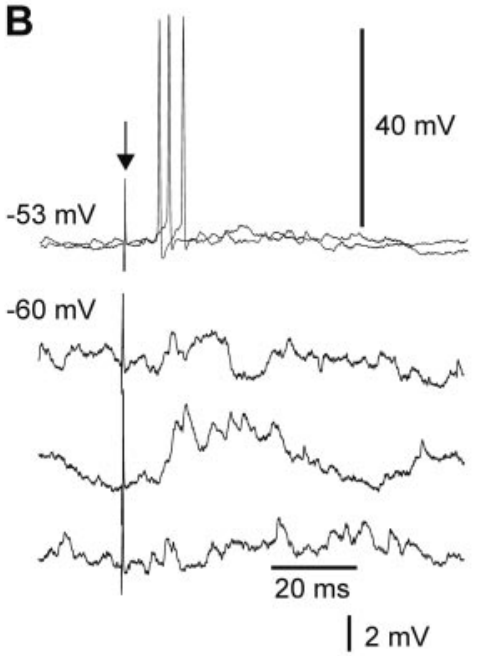

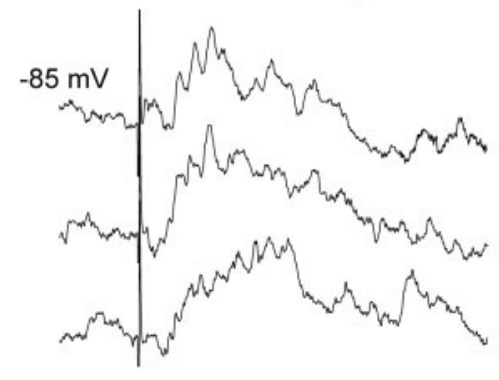

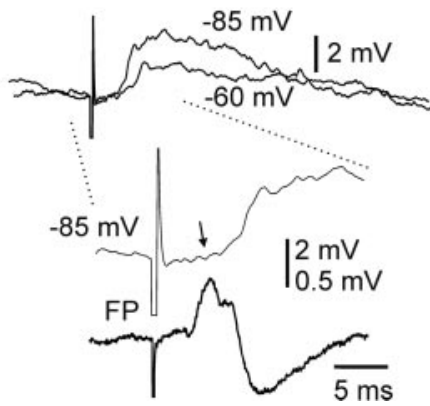

C

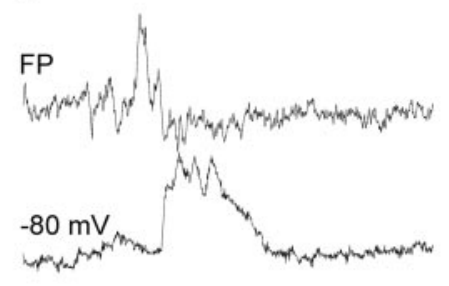

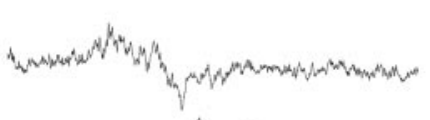

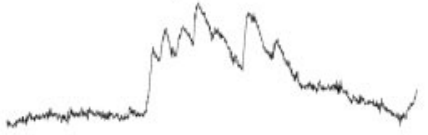

$0.3 \mathrm{mV}$ $6 \mathrm{mV} \quad 20 \mathrm{~ms}$

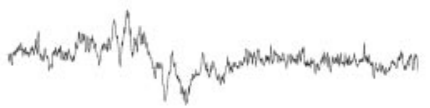

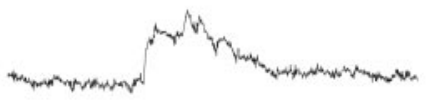

D

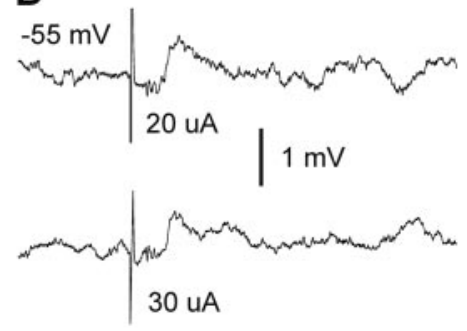

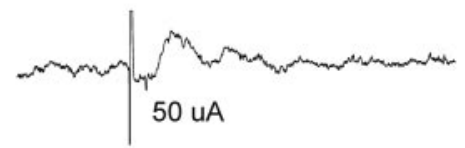

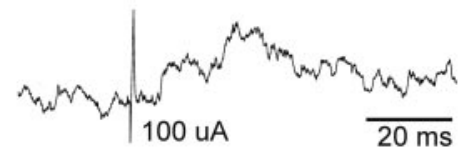
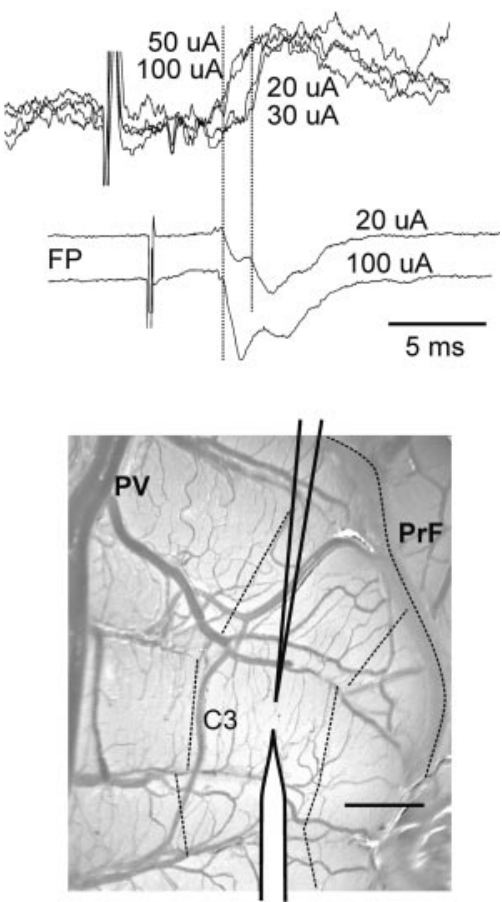

Figure 3. CF-evoked and -related responses. $A$, Schematic circuit diagram highlighting the CF input to interneurons. $B$, Responses evoked at different membrane potentials in the same cell by stimulation in the inferior olive at $100 \mu \mathrm{A}$ (arrow). Superimposed sweeps in the bottom compare averaged responses evoked at $-60 \mathrm{and}-85 \mathrm{mV}$. The response evoked at $-85 \mathrm{mV}$ is expanded and compared with the surface FP recorded nearby in the same sagittal plane. C, Spontaneous bursts of intracellular activity and their relationship to simultaneous surface field potentials recorded nearby $(\sim 0.2 \mathrm{~mm}$ away in the same sagittal plane). D, Averaged (F responses evoked from the inferior olive at indicated stimulation intensities. In bottom traces, the evoked responses are superimposed and compared with the FP, evoked at 20 and $100 \mu \mathrm{A}$, and were recorded simultaneously and at the same depth as the recorded cell. Note that the FP recording is shifted to the right to illustrate the parallel latency shifts between the FP and the intracellular response. The main part of the field potential shifted to shorter onset latency times between 30 and $50 \mu \mathrm{A}$. Note that local CF responses have a positive polarity at the surface but a negative polarity in the molecular layer where they are generated (Eccles et al., 1967). Inset illustrates the location in the cerebellum of the whole-cell recording pipette (bottom) and the metal microelectrode recording field potentials. Also shown are the borders of the (3 zone, the primary fissure (PrF), and the paravermal vein (PV). Scale bar, $1 \mathrm{~mm}$.

input in the in vivo preparation (Paré et al., 1998). The time constants were $2.4 \pm 1.0 \mathrm{msec}(n=16$; range, $1-4 \mathrm{msec})$. As in extracellular recordings (Ekerot and Jörntell, 2001, 2003; Jörntell and Ekerot, 2002), the spontaneous spike firing was irregular. Spontaneous spike firing appeared to depend primarily on the occurrences of large EPSP-like events (Fig. $1 E$ ).

\section{Activity evoked by PFs}

Electrical stimulation of on-beam PFs evoked depolarizing events that, at threshold stimulation, fluctuated greatly (Fig. $2 A, B$ ), similar to what has been reported for PF-EPSCs in interneurons in vitro (Chen and Regehr, 1999). Increased stimulation intensity produced larger and less variable responses, which eventually 
A

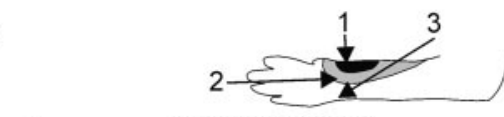

1

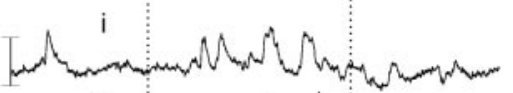

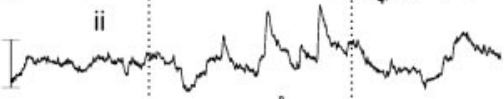

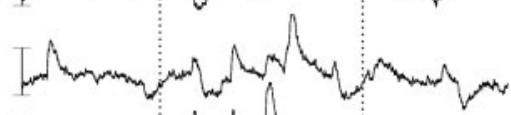

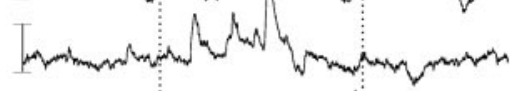

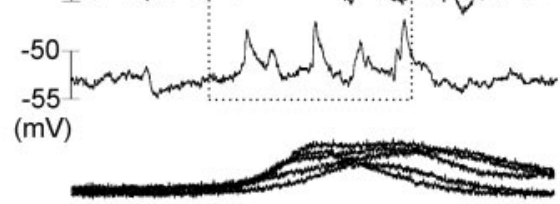

B

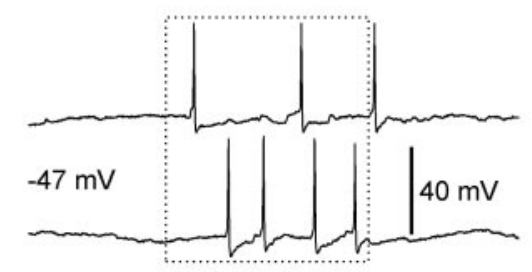

D

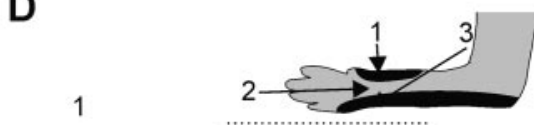

1

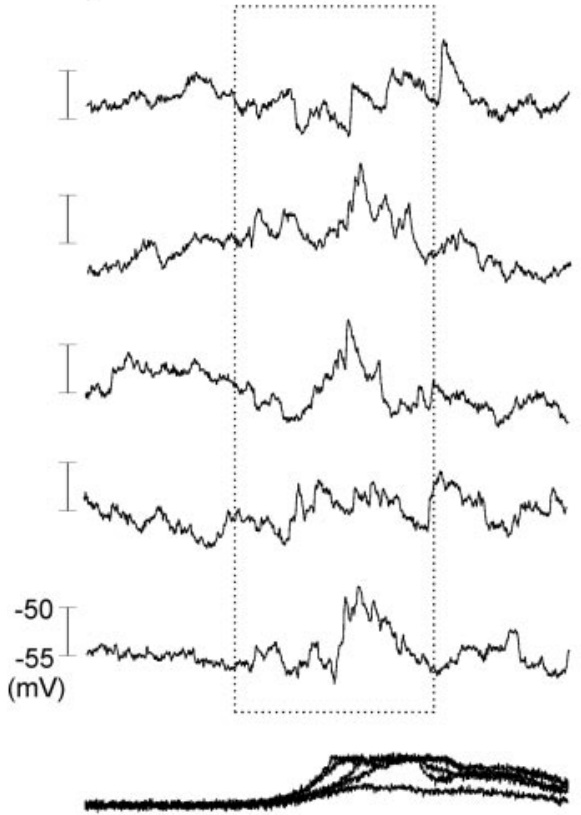

E



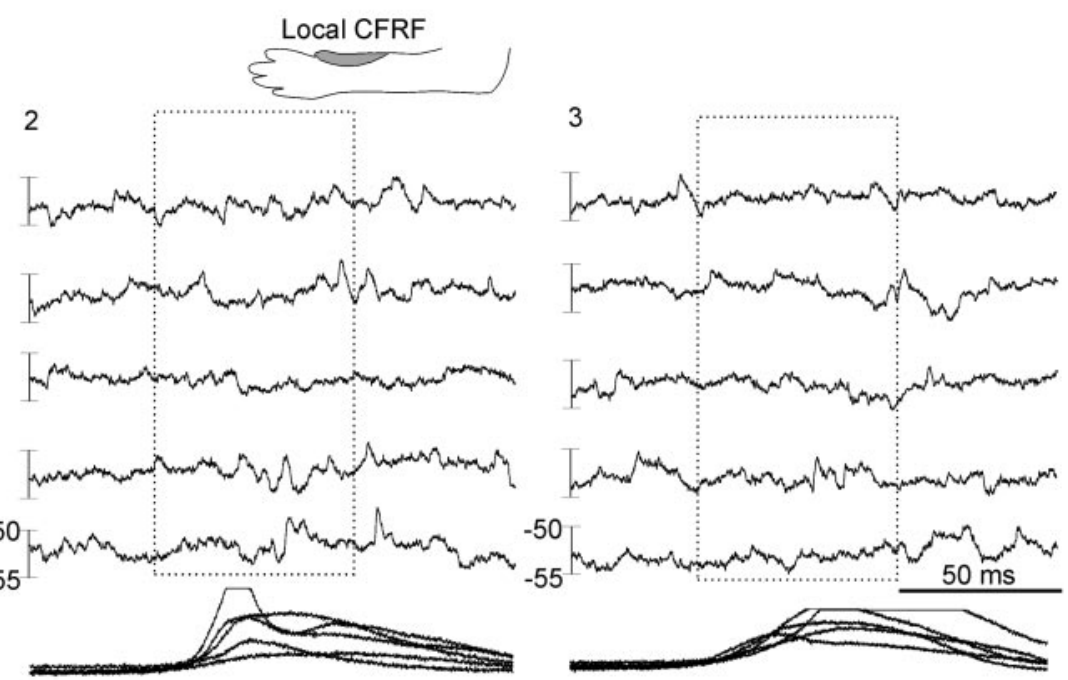

C
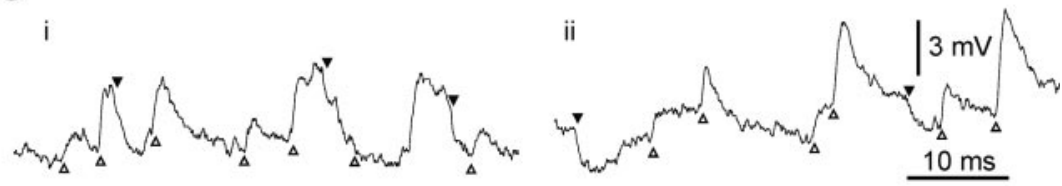
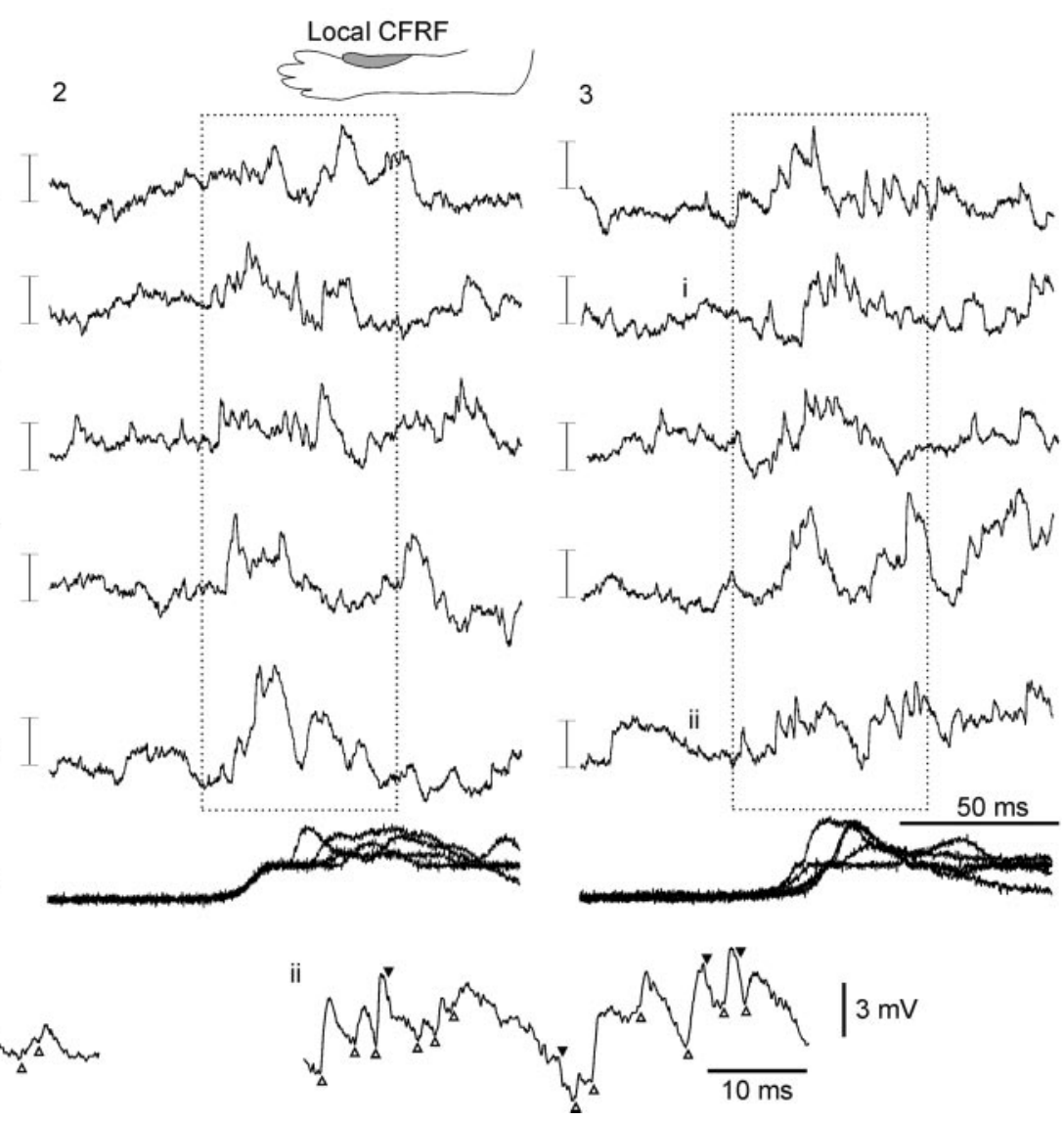

Figure 4. Manual stimulation before and after conjunctive PF-CF stimulation. A, Responses evoked by skin strokes in a normal interneuron. The responses evoked from three different skin sites (1-3) are illustrated by five consecutive raw data sweeps each. The location of these skin sites is shown in relation to the receptive field estimated from the responses evoked from these and six other stimulation sites on forelimb. Degree of gray shading in the receptive field indicates degree of EPSP driving. On another forelimb outline, the local (F receptive field (CFRF) is indicated. Bottom traces are the signal from the strain gauge device, indicating that approximately the same amount of stimulation was applied to the three skin sites. In some cases, this signal reached the voltage limit of the analog-to-digital converter. B, Spike response of the same interneuron to stimulation in site 1 with 0 pA bias current. C, Blowups from two raw data sweeps (i, iii), allowing a detailed inspection of the evoked responses. Open and filled triangles indicate the onsets of substantial putative EPSPs and IPSPS, respectively. D, E, Display as in $A$ and $C$ but for another interneuron after conjunctive PF-CF stimulation. 
A

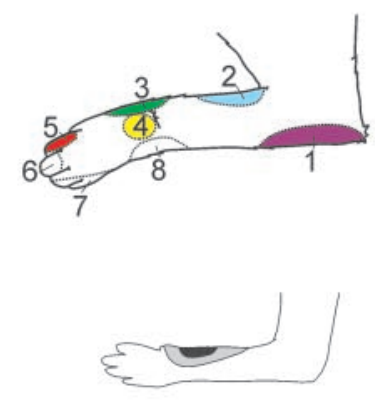

C

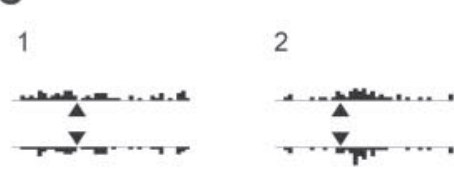

5

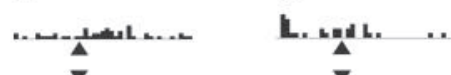

‥

D

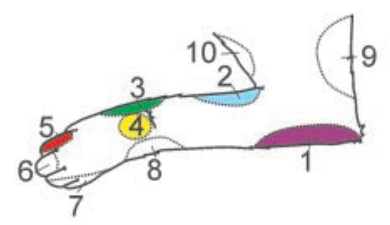

F

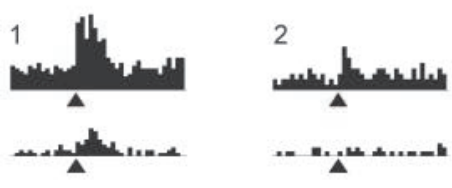

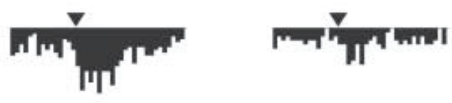

6 年

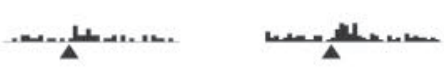

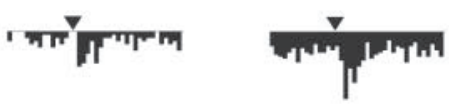
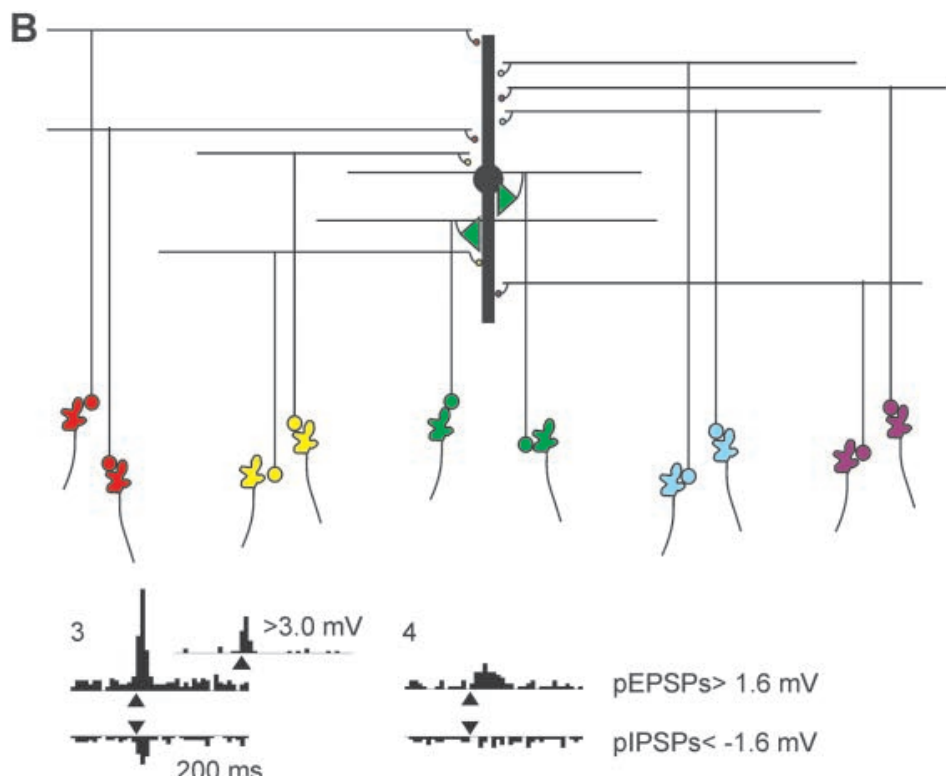

\begin{tabular}{l|l}
7 & $1 \mathrm{PSP} / \mathrm{stim}$
\end{tabular}

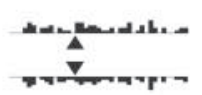

$8 \quad \mathrm{HH}$

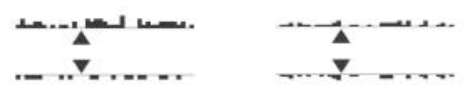

E



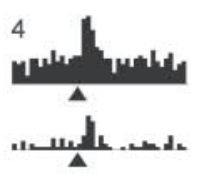

5

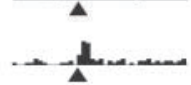$$
\text { its.t. }
$$

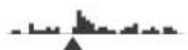

pEPSPs $>1.9 \mathrm{mV}$ PEPSPs $>3.0 \mathrm{mV}$

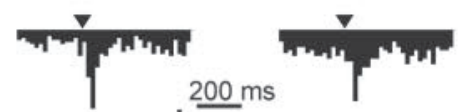
1 PSP/stim

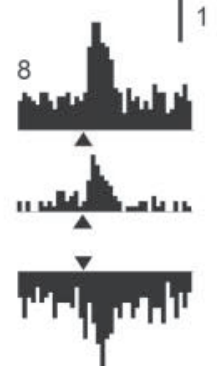

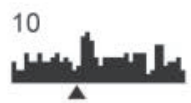

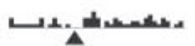

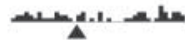

T

$\frac{v}{1-1 / 1+1 / 4}$
M 1 pIPSPs $<-1.9 \mathrm{mV}$ coFL

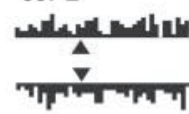


produced a spike. Events similar to those evoked at threshold PF stimulation occurred also as spontaneous events (Fig. 2D,G). The variable behavior at threshold stimulation, the time courses, and the amplitude changes across different levels of membrane potential (Fig. 2C) are consistent with these events being EPSPs and IPSPs. Generally, the maximal peak amplitudes for unitary, spontaneous EPSPs ranged between 5.2 and $7.5 \mathrm{mV}$. The time-topeaks were $0.5-1.3 \mathrm{msec}$, and there was no apparent relationship between the value of this parameter and specific EPSP amplitudes. The amplitude distribution of spontaneous EPSPs decayed roughly exponentially toward higher peak amplitudes (Fig. $2 E, F)$. For unitary spontaneous IPSPs, maximal peak amplitudes were generally somewhat smaller (absolute values), the time-topeak values were overall $\sim 1 \mathrm{msec}$ longer, and they had a longer decay time than EPSPs (Fig. 2D-G, Table 1).

\section{Activity evoked by CFs}

To study the CF input in isolation, free of contamination from MF-PF input, we had to stimulate CFs directly at their origin in the inferior olive. Before intracellular recordings commenced, we searched with metal microelectrodes for points in the inferior olive in which thresholds for evoking CF field potentials (FPs) in the cerebellar region of interest were at a minimum $(4-20 \mu \mathrm{A})$. The response in interneurons to inferior olivary stimulation was typically strong but also highly variable when the interneurons were kept at a membrane potential just below threshold for spike firing (Fig. 3B). At more hyperpolarized levels, when spontaneous IPSPs were indiscernible (at $-85 \mathrm{mV}$ ), the same stimulation more consistently produced a strong depolarizing response. The averaged response at this membrane potential was initiated within $1 \mathrm{msec}$ of the onset of the local CF field potential, but it usually started to grow rapidly only $2-3 \mathrm{msec}$ later. Furthermore, the CF response had a much longer duration $(20-60 \mathrm{msec})$ than PF EPSPs (Fig. 3B). We also found a coupling between spontaneous CF field potentials and the occurrence of depolarizations reminiscent of the evoked CF responses (Fig. 3C). The amplitude of the averaged CF response appeared to be independent of the olivary stimulation intensity, but when the intensity was lowered so that the peak of the CF field potential shifted toward longer response latencies, the response latency of the averaged CF response in the interneuron shifted in parallel (Fig. 3D).

\section{Cutaneous synaptic input before and after conjunctive $\mathrm{PF}-\mathrm{CF}$ stimulation}

After conjunctive PF burst and CF stimulation, the synaptic activity, compared with normal interneurons, was changed markedly in interneurons recorded on-beam for the stimulated PFs. This effect was clearly present also in recordings obtained $>3 \mathrm{hr}$ after the protocol, confirming our previous observations in extracellular recordings that the induced receptive field changes last at least several hours (Jörntell and Ekerot, 2002). In contrast, a corresponding stimulation protocol, but omitting the PF burst stimulation (CF alone), had no effect on the receptive fields of interneurons $(n=10)$. The PF-alone protocol (i.e., PF burst protocol without CF activation) was not tested in the present series, because we have shown previously it to be without effect on the receptive fields of normal (naive) interneurons recorded extracellularly (Jörntell and Ekerot, 2002).

\section{Manual skin stimulation}

Peripheral driving of EPSPs and IPSPs was investigated using rapid, light strokes across a restricted area of the skin, a very effective stimulation for driving the spikes of interneurons within the C3 zone (Ekerot and Jörntell, 2001, 2003; Jörntell and Ekerot, 2002). Just like extracellular spikes, in normal (naive) interneurons, the EPSPs were driven nearly exclusively from one specific skin area $(n=10)$, overlapping the receptive field of CFs terminating on local Purkinje cells (Fig. 4A). Individual responses evoked from this specific skin area lasted for the duration of the stimulation $(\sim 50 \mathrm{msec})$ and consisted of remarkably few distinct EPSPs (four to eight). The number of depolarization peaks (two to five) corresponded well to the number of spikes evoked in the cells when the hyperpolarizing current was removed (Fig. $4 B$ ) as well as in extracellular recordings from these interneurons using the same type of skin stimulation (Jörntell and Ekerot, 2002). Also, there was an apparent lack of summation of small EPSPs to create slow buildups of membrane depolarization. Hence, the large, unitary EPSPs seemed to be the only substantial contributors to the cutaneous driving of the cell (Fig. 4C).

In interneurons after conjunctive $\mathrm{PF}-\mathrm{CF}$ stimulation, the synaptic input evoked by cutaneous stimulation was different in many ways $(n=4)$ (Fig. $4 D, E$, Table 1$)$. First, EPSPs and IPSPs were driven from the entire forelimb and not from a specific skin area as in normal interneurons. Second, the number of evoked EPSPs per stroke was substantially higher, and both small and large EPSPs were activated from the entire forelimb. In this case, slow depolarizations that would be expected from the summation of a great number of small EPSPs occurred frequently.

To compare the responses evoked from different skin sites, we constructed peristimulus histograms of EPSPs and IPSPs (Fig. 5). In normal interneurons, EPSPs were driven from a small skin area only (Fig. $5 C$, sites $2-4$ ). EPSPs $>3.0 \mathrm{mV}$ only occurred as evoked responses from site 3 (inset histogram). Adjacent skin areas could provide weak driving of medium-sized EPSPs (sites 2 and 3), hence forming a peripheral part of the receptive field with a lower sensitivity to skin contact. In contrast, in interneurons recorded after conjunctive $\mathrm{PF}-\mathrm{CF}$ stimulation (Fig. 5F), EPSPs and IPSPs of all amplitudes were driven from all skin sites on the ipsilateral forelimb (but not from any other part of the body skin). Note also that the background frequencies of spontaneous EPSPs tended to be higher in interneurons after conjunctive $\mathrm{PF}-\mathrm{CF}$ activation (Table 1). The increased spontaneous PSP frequency is also visible as a change in the baseline levels preceding stimulation onset in the histograms (Fig. 5, compare $C, F$ ).

Figure 5. Cutaneous activation of PSPs before and after conjunctive PF-CF stimulation. $A$, Stimulated skin sites and receptive field of normal interneuron. $B$, Schematic circuit diagram illustrating the topographical distribution of mossy fibers with specific receptive fields and the transmission of their information via granule cells-PFs to an unconditioned interneuron. Large, triangular PF synapses indicate that the information is transmitted to the interneuron, whereas small dot synapses indicate that it is not (Jörntell and Ekerot, 2002). Color coding of mossy fibers- granule cells-PF synapses refers to skin sites in A. The somatotopic sequence shown is typically found for mossy fibers in the medial half of the (3 zone (Garwicz et al., 1998). C, Peristimulus histograms of EPSPs and IPSPs (upside-down histograms) (pEPSPs and pIPSPs, respectively) evoked from skin sites $1-8$ and ipsilateral hindlimb (iHL). Data display is -300 to $+500 \mathrm{msec}$ relative to the time of skin contact, indicated by arrowheads. Inset histogram for site 3 shows that EPSPs with peak amplitudes of $>3.0 \mathrm{mV}$ were driven from this site only. $D-F$, Similar display as in $A-C$ but for another interneuron after conjunctive PF-CF stimulation. Note that in this case, PSPs as well as EPSPs $>3.0 \mathrm{mV}$ were driven from all skin sites on the ipsilateral forelimb. coFL, Contralateral forelimb. Same two cells as in Figure 4. 

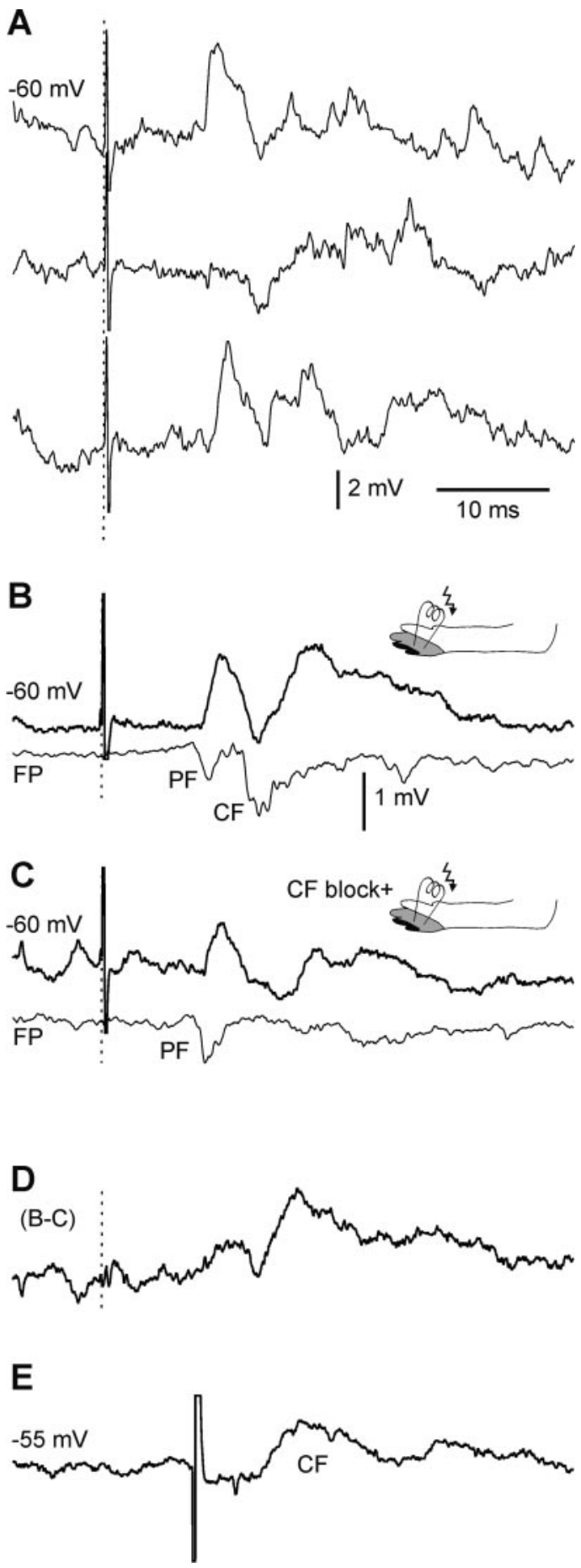

Figure 6. Responses to electrical skin stimulation in normal interneurons. $A$, Responses evoked in an interneuron by electrical skin stimulation shown as raw data sweeps to illustrate the variability of the response. Dotted vertical line indicates time of electrical shock. B, Averaged response of the same interneuron and the field potential recorded in the molecular layer nearby (same cell as in Fig. 3D).C, Averaged response evoked by skin stimulation when transmission of CF impulses was blocked by a $200 \mathrm{~Hz}$ double-shock stimulation in the inferior olive preceding the skin-evoked response by $40 \mathrm{msec}$. Note the complete block of CF responses in the field potential recording and that the PF field response appeared unaffected. D, Subtraction of sweep C from sweep B. E, The averaged CF response evoked by stimulation in the inferior olive at $50 \mu \mathrm{A}$.

\section{Electrical skin stimulation}

In the $\mathrm{C} 3$ zone in our preparation, electrical skin stimulation evokes a compound response in which the onset times of PF and CF synaptic input can be deduced from local field potentials. In normal interneurons, electrical stimulation of the skin evoked an initial strong depolarization at about the same time as the onset of the PF field potential, followed by a hyperpolarization, and soon after the onset of the local CF field potential, a strong and more long-lasting depolarization (Fig. 6A,B). Using a conditioning stimulation in the inferior olive, the CF input was blocked and the peripherally evoked response consisted of PF input only (Fig. 6C). Hence, the PF response lasted for at least $20 \mathrm{msec}$ after the onset of the PF field potential. When the pure PF response was subtracted from the unconditioned response in Figure $6 B$, mainly the CFevoked response remained (Fig. $6 D$ ), and this was found to correspond well in both time course and amplitude to the averaged $\mathrm{CF}$ response evoked from the inferior olive (Fig. $6 E$ ).

In interneurons after conjunctive PF-CF stimulation, electrical stimulation in the induced receptive field evoked a slower and less steep response than the same stimulation in the original receptive field (Fig. 7A). In addition, the first evoked EPSP generally occurred earlier and had a larger peak amplitude when the original receptive field was stimulated (Fig. $7 C$ ). The mean $( \pm \mathrm{SD})$ response latency of the first evoked EPSP was $9.34 \pm 0.62$ and $10.15 \pm 0.89 \mathrm{msec}$, respectively. These findings could be explained by the anatomical organization of the cortical circuitry. MF input from the original receptive field terminates right beneath the interneurons, whereas MF input from induced receptive fields has to travel a longer distance in the PFs before it reaches the interneuron (Fig. 7D). The conduction velocity of PFs has been estimated to be $0.3 \mathrm{~mm} / \mathrm{msec}$ (Eccles et al., 1967), so the difference in travel distance for the two inputs should be $\sim 0.3$ $\mathrm{mm}$. This is in fair agreement with the known topographical distribution of MFs activated from the two respective skin areas (Garwicz et al., 1998).

\section{Discussion}

Using controlled manual stimulation of small skin sites, we found the synaptic integration of cutaneous PF input in normal interneurons to be very simple and entirely dominated by a few large EPSPs driven from the same small area of the skin. In contrast, after conjunctive PF-CF stimulation, a wide range of small to large EPSPs could be evoked from any skin area on the forelimb. This is the first demonstration that conjunctive PF-CF activation leads to a potentiation of synaptic PF input to interneurons. The CF input, which is crucial for this potentiation but has not been described before, was a relatively slow and long-lasting response.

Remarkably, the peripheral input to normal (naive) interneurons consisted of as few as two to eight substantial EPSPs, and slower depolarizations that could result from summation of smaller EPSPs were widely lacking. Because cutaneously activated MFs fire intense bursts of action potentials during the type of manual stimulation that was used here (Garwicz et al., 1998; Ekerot and Jörntell, 2001), it is likely that at least some granule cells fire more than one spike per stimulation. Hence, the skindriven output of molecular layer interneurons might be controlled by as few as two granule cells (one granule cell seems unlikely because of the irregular intervals between some EPSPs, as shown in the individual traces in Fig. $4 A, C$ ). Interestingly, our previous estimation, on the basis of receptive field analysis in extracellular recordings and the knowledge of the available pool of MF receptive field types, was that only $\sim 2-3 \%$ of the cutaneously activated $\mathrm{PF}$ synapses on interneurons are electrically active (Ekerot and Jörntell, 2001, 2003; Jörntell and Ekerot, 2002). If interneurons receive a few hundred PF synapses (Carter and Regehr, 2002), and if approximately half of these PF synapses are activated by noncutaneous input such as cerebrocerebellar input, 
A
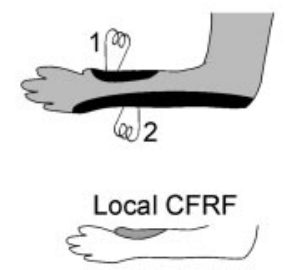

B

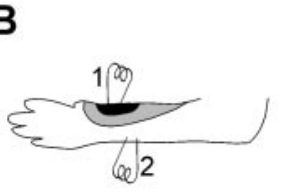

C
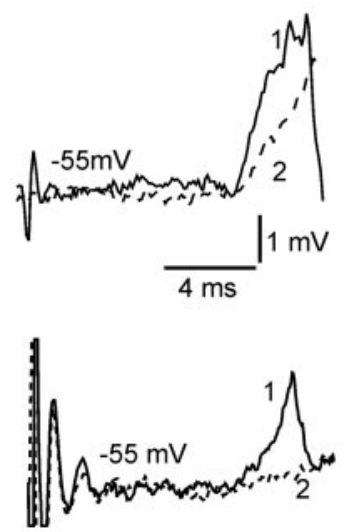

D

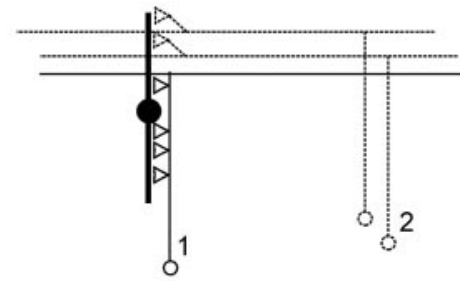

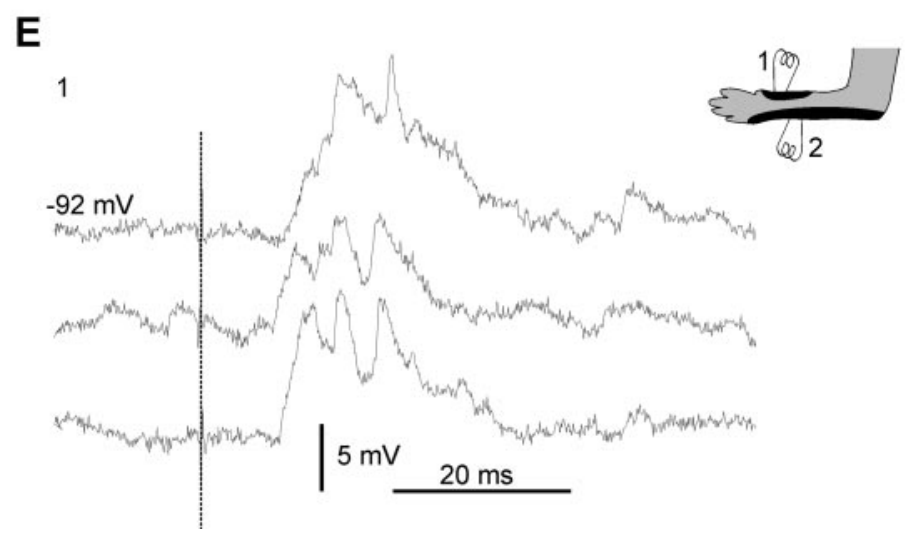

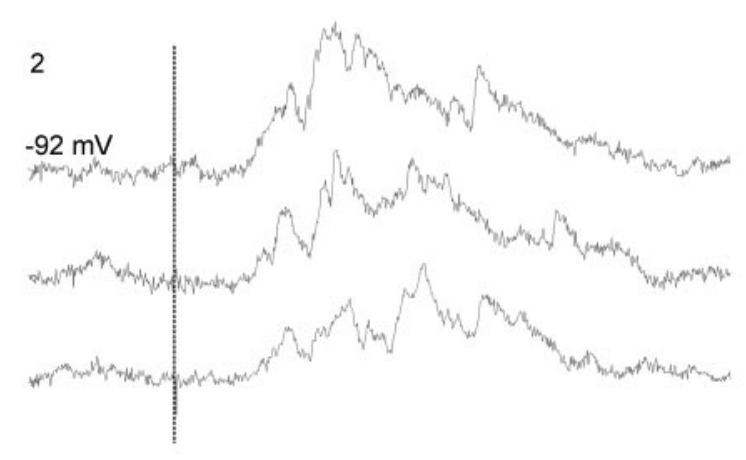

Figure 7. Responses to electrical skin stimulation in original and induced receptive fields. $A$, The onset of the averaged responses to an electrical shock in original [1, similar location on the skin as the local CF receptive field (CFRF), black trace] and induced (2, dashed trace) receptive fields in an interneuron after conjunctive PF-CF stimulation. B, Averaged responses in a normal interneuron to electrical shock within the same skin sites (i.e., for this cell, within and outside the receptive field). C, Scatter plot of the amplitude and onset latency for the first EPSP evoked by electrical shock to the original (dots) and induced (crosses) receptive fields for 23 consecutive responses. D, Schematic circuit diagram illustrating the pathways through the cerebellar cortex for input from the original and induced receptive fields, respectively. Note that the responses analyzed in $A-C$ are pure PF responses because they occurred before the time of arrival of the evoked $C F$ input (which, in the (3 zone, have a response latency of $13 \mathrm{msec}$ or more) (compare Fig. 6B). E, Full raw data responses evoked by electrical shocks in the original (left) and induced (right) receptive fields. Dotted vertical line indicates time of electrical shock. In these sweeps, the neuron was hyperpolarized below the reversal potential for IPSPs to better illustrate the full length of the responses.

these estimations indicate that the cutaneous input to an interneuron may well derive from as few as two to three granule cells. From skin areas adjacent to the center of the receptive field, weaker activation of medium-sized EPSPs was evoked. Hence, activation of EPSPs may be through distinct groups of granule cells: large EPSPs are evoked only from granule cells with receptive fields that fit precisely in the center of the local CF receptive field, whereas medium-sized EPSPs can be activated from granule cells with receptive fields that partly overlap but less precisely match the local CF receptive field. Stronger EPSPs from PFs with receptive fields that better fit with the CF receptive field would be an expected effect if conjunctive PF-CF activation leads to strengthening of PF synapses in the behaving animal. This is because receptive field similarity should be an important factor in determining the timing of PF and CF activation. Outside the restricted receptive field of normal interneurons, no EPSPs were evoked.

The rate of both evoked and spontaneous IPSPs displayed a remarkable parallelism to the rate of EPSPs, regardless of whether the excitatory receptive field of the interneuron was normal, and restricted or included the entire forelimb skin as after potentiation of the PF input (Fig. 5, Table 1). Because interneurons with similar excitatory receptive fields are normally found within the same parasagittal plane (Ekerot and Jörntell, 2001, 2003; Jörntell and Ekerot, 2002), and because their axons ramify mainly locally and parasagittally (Palay and Chan-Palay, 1974), this parallelism can be explained by mutual inhibition between molecular layer interneurons sharing the same excitatory input. Therefore, cerebellar interneurons share many features with interneurons in the neocortex, in which mutual inhibition and intracellular communications between local interneurons have been reported recently (Galaretta and Hestrin, 1999, 2001; Gibson et al., 1999), as has a very strong influence on spike firing by single excitatory afferents (Galaretta and Hestrin, 2001). Also, the strong tendency for interneurons with mutual electrical and inhibitory synaptic connections to fire in synchrony, as observed in vitro (Beierlein et al., 2000; Galaretta and Hestrin, 2001), should be even more pronounced for cerebellar interneurons in vivo, because they also receive excitatory input from the same small area of the skin (Ekerot and Jörntell, 2001, 2003).

Intracellular CF responses in cerebellar interneurons have not been described previously and were therefore of key interest in the present study. Surprisingly, CF activation resulted in longlasting, complex responses with similar characteristics. The latency of the main part of the response was 2-4 msec longer than that of the CF field potentials and CF responses in nearby Purkinje cells. Only a very small depolarization occurred at latencies compatible with a direct synaptic response (i.e., within $1 \mathrm{msec}$ 
after the onset of the CF field potential), allowing for a possible extra delay for impulses to travel through the thin collaterals innervating the interneuron (Sugihara et al., 1999). Several observations make it unlikely that the response evoked from the inferior olive was attributable to activation of mossy fiber pathways, as follows. (1) Care was taken to place the stimulating electrode at the location in the inferior olive with the lowest possible threshold $(<20 \mu \mathrm{A})$ to evoke CF field potentials in the recording area. Different stimulation intensities did not change the main features of the response (Fig. 3D). (2) A parallel shift in onset latencies was observed for the main part of the CF field potential and the evoked response in the interneuron (Fig. 3D). (3) Coupling between the spontaneous CF field potentials and intracellular depolarizations resembled the responses evoked from the inferior olive (Fig. 3C). (4) When the CF input was temporarily blocked, the intracellular response evoked by electrical skin stimulation was reduced by one component, which resembled the CF response evoked from the inferior olive (Fig. 6).

The response mechanism of the interneuronal CF response is still unclear. Given the crucial role of CFs in the plasticity of PF input to interneurons (Jörntell and Ekerot, 2002), the slow, longlasting CF response may reflect the process by which the tiny CF synapses influence PF synapses throughout the interneuron.

After a protocol with repeated PF burst stimulation paired with CF activation, EPSPs could be evoked from the entire forelimb skin, and there seemed to be an accompanying increase in the background frequency of EPSPs (Table 1), just as for extracellular spikes (Jörntell and Ekerot, 2002). As shown previously, MF and CF receptive fields are unchanged after this protocol (Jörntell and Ekerot, 2002). In addition, because the protocol induces opposite effects in the PF receptive fields of interneurons and Purkinje cells (expansion and shrinkage, respectively) (Jörntell and Ekerot, 2002), effects on granule cell receptive fields are ruled out. Also, because MFs terminating in the vicinity of one another have similar specific receptive fields, plastic changes in MF synapses on granule cells could not result in any radically different receptive fields in granule cells or interneurons (Garwicz et al., 1998; Ekerot and Jörntell, 2003). Because we now observe that EPSPs, and not only extracellular spikes, are driven from skin areas from which no such input is normally detected, the conjunctive $\mathrm{PF}-\mathrm{CF}$ stimulation must have induced a potentiation of previously ineffective PF synapses. Therefore, this is the first demonstration of a CF-induced PF long-term potentiation (LTP) in interneurons. It is also a confirmation of our previous suggestion that a large proportion ( $~ 95 \%$; see above) of the cutaneously activated PF synapses on interneurons are normally electrically silent or strongly depressed. Using a different approach, also a large proportion of PF synapses ( $\sim 85 \%)$ on Purkinje cells have been found to be electrically silent (Isope and Barbour, 2002).

The establishment and maintenance of the normal PF receptive fields of interneurons is likely to depend at least partly on a natural form of CF-induced PF LTP that should be activated in the behaving animal during development and adult life. Only PF synapses activated simultaneously with the local CF should be potentiated, whereas PF synapses that are activated at other times are instead likely to undergo depression, as suggested experimentally (Jörntell and Ekerot, 2002). The intracellular analysis in normal interneurons confirmed that there is indeed a small privileged set of PF synapses that alone are responsible for the cutaneous activation of EPSPs. After experimentally induced PF LTP, their privileged status vanished when the PF synaptic input from other skin areas increased remarkably. The fact that EPSPs of any size could be evoked from any skin area after PF LTP leads to the question of what determines the size of EPSPs for potentiated PF synapses. A speculative answer might be that silent PF synapses are in different states of downregulation depending on the history of their synaptic strength during development and adult life. PF synapses that evoke EPSPs with large amplitudes after potentiation might have been potentiated strongly in previous phases of the lifetime of the animal.

In many theories of cerebellar function, the CF input is believed to be a signal for performance errors that could lead to remodeling of the cerebellar circuits through synaptic plasticity (Gilbert and Thach, 1977; Ito, 1984, 2001; Andersson and Armstrong, 1987; Kitazawa et al., 1998). Interestingly, the motor output regulated by the Purkinje cells is specific to their CF receptive fields so that activation of an interneuron through skin contact with an external object would result, via a reduction in Purkinje cell inhibition in the deep cerebellar nucleus, in a muscle activation that tended to withdraw that skin area from the object (Ekerot et al., 1995; Ekerot and Jörntell 2001, 2003). Because the local CFs and interneurons normally have the same receptive fields, the primary role of the interneurons in an adapted cerebellar network may be to respond as fast as possible when the local CF is about to be activated and to prevent that activation by initiating a counteractive movement. For cerebellar function in general, interneurons may have the role of learning to predict conditions that could lead to CF activation and could prevent, by their inhibitory control of the efferent Purkinje cell, that condition through efferent commands elicited by disinhibition in the deep cerebellar nuclei. This property would make the interneurons and the CF-induced PF LTP suitable as an explanation for the behavioral effects during, for example, classical conditioning, one of the behavioral adaptations for which a cerebellar role is best documented (Thompson and Kim, 1996).

\section{References}

Andersson G, Armstrong DM (1987) Complex spikes in Purkinje cells in the lateral vermis (b zone) of the cat cerebellum during locomotion. J Physiol (Lond) 385:107-134.

Beierlein M, Gibson JR, Connors BW (2000) A network of electrically coupled interneurons drives synchronized inhibition in neocortex. Nat Neurosci 3:904-910.

Brand S, Dahl AL, Mugnaini E (1976) The length of parallel fibers in the cat cerebellar cortex. An experimental light and electron microscopic study. Exp Brain Res 26:39-58.

Callaway JC, Lasser-Ross N, Ross WN (1995) IPSPs strongly inhibit climbing fiber-activated $\left[\mathrm{Ca}^{2+}\right]_{\mathrm{i}}$ increases in the dendrites of cerebellar Purkinje neurons. J Neurosci 15:2777-2787.

Carter AG, Regehr WG (2002) Quantal events shape cerebellar interneuron firing. Nat Neurosci 5:1309-1318.

Chavas J, Marty A (2003) Coexistence of excitatory and inhibitory GABA synapses in the cerebellar interneuron network. J Neurosci 23:2019-2031.

Chen C, Regehr WG (1999) Contributions of residual calcium to fast synaptic transmission. J Neurosci 19:6257-6266.

De Camilli P, Miller PE, Levitt P, Walter U, Greengard P (1984) Anatomy of Purkinje cells in the rat determined by a specific immunohistochemical marker. Neuroscience 11:761-817.

Eccles JC, Ito M, Szentágothai J (1967) The cerebellum as a neuronal machine. Berlin: Springer.

Ekerot CF, Oscarsson O (1981) Prolonged depolarization elicited in Purkinje cell dendrites by climbing fibre impulses in the cat. J Physiol (Lond) 318:207-221.

Ekerot CF, Jörntell H (2001) Parallel fibre receptive fields of Purkinje cells and interneurons are climbing fibre-specific. Eur J Neurosci 13:1303-1310.

Ekerot CF, Jörntell H (2003) Parallel fiber receptive fields: a key to understanding cerebellar operation and learning. Cerebellum 2:101-109.

Ekerot CF, Jörntell H, Garwicz M (1995) Functional relation between cor- 
ticonuclear input and movements evoked on microstimulation in cerebellar nucleus interpositus anterior in the cat. Exp Brain Res 106:365-376.

Galaretta M, Hestrin S (1999) A network of fast-spiking cells in the neocortex connected by electrical synapses. Nature 402:72-75.

Galaretta M, Hestrin S (2001) Spike transmission and synchrony detection in networks of GABAergic interneurons. Science 292:2295-2299.

Garwicz M, Jörntell H, Ekerot CF (1998) Cutaneous receptive fields and topography of mossy fibres and climbing fibres projecting to cat cerebellar C3 zone. J Physiol (Lond) 512:277-293.

Gibson JR, Beierlein M, Connors BW (1999) Two networks of electrically coupled inhibitory neurons in neocortex. Nature 402:75-79.

Gilbert PFC, Thach WT (1977) Purkinje cell activity during motor learning. Brain Res 128:309-328.

Hamill OP, Marty A, Neher E, Sakmann B, Sigworth FJ (1981) Improved patch-clamp techniques for high-resolution current recording from cells and cell-free membrane patches. Pflügers Arch 391:85-100.

Häusser M, Clarke BA (1997) Tonic synaptic inhibition modulates neuronal output pattern and spatiotemporal synaptic integration. Neuron 19:665-678.

Isope P, Barbour B (2002) Properties of unitary granule cell-Purkinje cell synapses in adult rat cerebellar slices. J Neurosci 22:9668-9678.

Ito M (1984) The cerebellum and neural control. New York: Raven.

Ito M (2001) Cerebellar long-term depression: characterization, signal transduction, and functional roles. Physiol Rev 81:1143-1195.

Jörntell H, Ekerot CF (2002) Reciprocal bi-directional plasticity of parallel fiber receptive fields in cerebellar Purkinje cells and their afferent interneurons. Neuron 34:797-806.

Kitazawa S, Kimura T, Yin PB (1998) Cerebellar complex spikes encode both destinations and errors in arm movements. Nature 392:494-497.

Kreitzer AC, Carter AG, Regehr WG (2002) Inhibition of interneuron firing extends the spread of endocannabinoid signaling in the cerebellum. Neuron 34:787-796.

Llinas R, Sugimori M (1980) Electrophysiological properties of in vitro Purkinje cell dendrites in mammalian cerebellar slices. J Physiol (Lond) 305:197-213.

Mann-Metzer P, Yarom Y (1999) Electrotonic coupling interacts with intrinsic properties to generate synchronized activity in cerebellar networks of inhibitory interneurons. J Neurosci 19:3298-3306.

Palay SL, Chan-Palay V (1974) Cerebellar cortex. Berlin: Springer.

Paré D, Shink E, Gaudreau H, Destexhe A, Lang EJ (1998) Impact of spontaneous synaptic activity on the resting properties of cat neocortical pyramidal neurons in vivo. J Neurophysiol 79:1450-1460.

Sugihara I, Wu H-S, Shinoda Y (1999) Morphology of single olivocerebellar axons labeled with biotinylated dextran amine in the rat. J Comp Neurol 414:131-148.

Sultan F, Bower JM (1998) Quantitative Golgi study of the rat cerebellar molecular layer interneurons using principal component analysis. J Comp Neurol 393:353-373.

Thompson RF, Kim JJ (1996) Memory systems in the brain and localization of a memory. Proc Natl Acad Sci USA 93:13438-13444. 